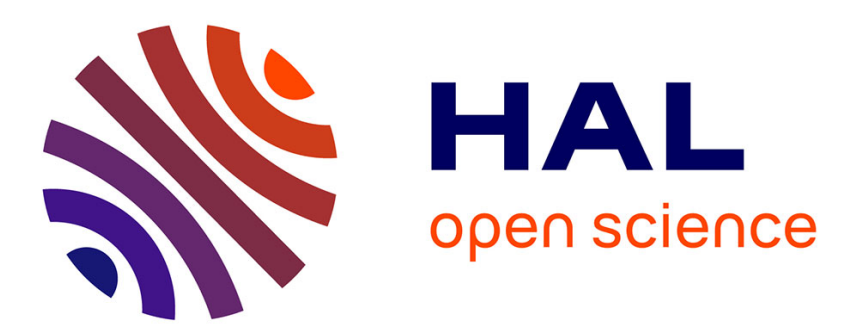

\title{
Magnetohydrodynamic self-propulsion of active matter agents
}

\author{
Alexander Zharov, Vanessa Fierro, Alain Celzard
}

\section{To cite this version:}

Alexander Zharov, Vanessa Fierro, Alain Celzard. Magnetohydrodynamic self-propulsion of active matter agents. Applied Physics Letters, 2020, 117 (10), pp.104101. 10.1063/5.0018692 . hal03043513

\section{HAL Id: hal-03043513 \\ https://hal.univ-lorraine.fr/hal-03043513}

Submitted on 19 Dec 2020

HAL is a multi-disciplinary open access archive for the deposit and dissemination of scientific research documents, whether they are published or not. The documents may come from teaching and research institutions in France or abroad, or from public or private research centers.
L'archive ouverte pluridisciplinaire HAL, est destinée au dépôt et à la diffusion de documents scientifiques de niveau recherche, publiés ou non, émanant des établissements d'enseignement et de recherche français ou étrangers, des laboratoires publics ou privés. 


\title{
Magnetohydrodynamic self-propulsion of active matter agents
}

\author{
Alexander Zharov, ${ }^{1,2}$ Vanessa Fierro, ${ }^{1}$ and Alain Celzard ${ }^{1,}$ a) \\ 1) Université de Lorraine, CNRS, IJL,F-88000 Epinal, France \\ ${ }^{2)}$ Institute for Physics of Microstructures, Russian Academy of Sciences, Nizhny Novgorod 603950, \\ Russia
}

Self-propelled particles, based on the magnetohydrodynamic acceleration of the surrounding fluid, are proposed for application as active matter agents. When the particle is resonantly excited by an electromagnetic wave, the scattered electric field induces currents in the surrounding fluid, which interact with the scattered magnetic field, thus producing a thrust. The time-averaged force is generally nonzero and can provide steady motion. Both orientation of the particle and direction of motion can be controlled by the radiation frequency and polarization. The concept has been studied analytically and through simulations showing its feasibility.

PACS numbers: 47.57.J-, 47.65.-d, 03.50.De

Active matter systems are composed of particles, called agents, which consume energy from the surroundings and convert it into motion. Initially inspired by biological systems, the study of active matter has become one of the fastest growing fields in soft condensed matter physics ${ }^{1,2}$. To date, with a variety of models describing the collective behavior of active matter $^{3}$, different types of biological and artificial agents have been studied and developed ${ }^{4}$.

Active matter agents use different self-propulsion mechanisms. The majority of biological micro-swimmers use either rotating or beating flagella to move through a liquid ${ }^{5,6}$. On the other hand, synthetic active particles commonly use other physical phenomena, including inhomogeneous catalysis of chemical reactions, or diffusiophoresis ${ }^{7-11}$, thermal inhomogeneity, or thermophoresis ${ }^{12-14}$, and mechanical motion induced by external fields ${ }^{15-17}$.

In this Letter, we propose a type of active matter agents that use magnetohydrodynamic (MHD) forces for propulsion. The agents are resonantly excited by an electromagnetic wave and produce crossed electric and magnetic fields in their own vicinity. The electric field generates currents in the surrounding fluid, which in turn interact with the magnetic field and produce the reaction force. We show that this force generally has a non-zero average over the period of oscillations and thus can provide steady motion of the agents. We have developed analytical models of the agent orientation in the external fields and the appearance of the MHD force. Finally, we have examined and verified the proposed concept through simulations. This study only focuses on the physical principle of propulsion and single-particle dynamics, leaving aside the collective behavior.

MHD propulsion ${ }^{18}$ is based on the acceleration of a conductive liquid (or plasma) using crossed electric and magnetic fields. Namely, the magnetic field interacts with the current induced by the electric field producing the Lorentz force, which, in turn, accelerates the liquid and generates the reaction force. In conventional MHD drives, the propulsion is produced by the interaction between the steady magnetic field and the direct current. However, oscillating current and magnetic field

\footnotetext{
a)Electronic mail: alain.celzard@univ-lorraine.fr
}

can also produce MHD thrust. In particular, the non-zero thrust is possible when the time-averaged Lorentz force does not vanish:

$$
\left\langle\mathbf{F}_{L}\right\rangle_{t}=\left\langle\int_{V}[\mathbf{j}(t) \times \mathbf{B}(t)] d V\right\rangle_{t} \neq 0,
$$

where $\left\langle\mathbf{F}_{L}\right\rangle_{t}$ is the time-averaged Lorentz force, $\mathbf{j}(t)$ is the current density and $\mathbf{B}(t)$ is the magnetic field. The integration goes over volume $V$, and angle brackets $\langle. .\rangle_{t}$ represent averaging over time $t$. For periodic processes, i.e., such that $\mathbf{j}(t), \mathbf{B}(t) \propto \exp (i \omega t)$, where $\omega$ is the angular frequency), Eq. (1) can be rewritten as:

$$
\left\langle\mathbf{F}_{L}\right\rangle_{T}=\int_{V} \operatorname{Re}\left[\mathbf{j}_{\omega} \times \mathbf{B}_{\omega}^{*}\right] d V \neq 0,
$$

where $T=2 \pi / \omega$ is the period of oscillations, Re stands for the real part, ${ }^{*}$ represents the complex conjugate, and $\mathbf{j}_{\omega}$ and $\mathbf{B}_{\omega}$ are the complex amplitudes of the current density and magnetic field, respectively. The cross product in Eq. (2) is non-zero when at least two conditions are satisfied: (i) there must be a region where the current density and magnetic field are non-zero and non-parallel, and (ii) the phase difference between them must be different from $\pi / 2$ or $3 \pi / 2$. The current density can be expressed as:

$$
\mathbf{j}=\sigma \mathbf{E},
$$

where $\sigma$ is the electrical conductivity of the fluid and $\mathbf{E}$ is the electric field. The high-frequency conductivity can be derived by considering the relations $\mathbf{j}=i \omega \mathbf{P}$ and $\varepsilon \varepsilon_{0} \mathbf{E}=\varepsilon_{0} \mathbf{E}+\mathbf{P}$, where $\mathbf{P}$ is the dipole moment density, $\varepsilon_{0}$ is the permittivity of vacuum, and $\varepsilon$ is the relative dielectric permittivity of the surrounding fluid. Hence, the conductivity can be expressed as:

$$
\sigma=i \omega \varepsilon_{0}(\varepsilon-1)
$$

In general, it is a complex number, $\sigma=\sigma^{\prime}+i \sigma^{\prime \prime}$, with the phase $\phi=\arctan \left(\sigma^{\prime \prime} / \sigma^{\prime}\right)$ defining the phase difference between the electric field $\mathbf{E}$ and the current $\mathbf{j}$ excited by it. It is noteworthy that the real part of the conductivity is defined by the imaginary part of the permittivity, i.e., the losses, while 
the imaginary part of the conductivity is defined by the real part of the permittivity. Thus, even a dielectric fluid with zero ionic conductivity has a non-zero imaginary high-frequency conductivity.

Let us consider a particle that can be represented by an $L C$-circuit: its equivalent electric scheme and possible designs are shown in Fig. 1. The capacitance and inductance of such a particle are defined by its shape, while the electromotive force (EMF) appears as a result of the interaction with an electromagnetic wave. In fact, a great variety of simpleconnected conducting particles are equivalent to the presented scheme. For instance, even a trivial metallic rod can be treated as an $L C$-circuit, as it has finite self-capacitance and selfinductance.

The magnetic field generated in the equivalent circuit presented in Fig. 1(a) is proportional to the current $I(t)=$ $I_{0} \exp (i \omega t)$ in the inductor. At the same time, the electric field between the capacitor plates is proportional to the capacitor charge $q(t)=q_{0} \exp (i \omega t)$. Taking Eqs. (2) and (3) into account, we can estimate the MHD force:

$$
\left\langle\left|\mathbf{F}_{L}\right|\right\rangle_{T}=\left|\int_{V} \operatorname{Re}\left(\sigma\left[\mathbf{E}_{\omega} \times \mathbf{B}_{\omega}^{*}\right]\right) d V\right| \propto \operatorname{Re}\left(\sigma q_{0} I_{0}^{*}\right) .
$$

Considering charge conservation, $I(t)=d q(t) / d t=i \omega q(t)$, we obtain:

$$
\left\langle\left|\mathbf{F}_{L}\right|\right\rangle_{T} \propto \operatorname{Re}\left(\left(\sigma^{\prime}+i \sigma^{\prime \prime}\right) i \omega q_{0}^{2}\right) \propto-\sigma^{\prime \prime} \omega q_{0}^{2}
$$

Expression Eq. (6) represents the integral body force acting on the liquid surrounding the particle. At the same time, the particle itself experiences the reaction force from the surrounding fluid, which has the same value and opposite direction. Indeed, one closed current system acts on another closed current system with the force:

$$
\mathbf{F}_{12}=-\frac{\mu_{0}}{4 \pi} \int_{V_{1}} \int_{V_{2}} \frac{\left(\mathbf{r}_{2}-\mathbf{r}_{\mathbf{1}}\right)\left(\mathbf{j}_{\mathbf{1}}\left(\mathbf{r}_{\mathbf{1}}\right) \cdot \mathbf{j}_{\mathbf{2}}\left(\mathbf{r}_{\mathbf{2}}\right)\right)}{\left|\mathbf{r}_{\mathbf{2}}-\mathbf{r}_{\mathbf{1}}\right|^{3}} d V_{1} d V_{2},
$$

where $V_{1}$ and $V_{2}$ are the volumes of the interacting systems, $\mathbf{r}_{1}$ and $\mathbf{r}_{2}$ are the radius vectors of the elements of the first and second systems, and $\mathbf{j}_{1}\left(\mathbf{r}_{1}\right)$ and $\mathbf{j}_{2}\left(\mathbf{r}_{2}\right)$ are the current densities at $\mathbf{r}_{1}$ and $\mathbf{r}_{2}$, respectively. It can be immediately seen that $\mathbf{F}_{21}=-\mathbf{F}_{12}$, which means that the first system acts on the second with a force with the same absolute value and the same backward direction as the second system acts on the first one. However, from the computational point of view, it is easier to find the body force density acting on the liquid than that acting on the particle.

It should be noted that, according to Eq. (6), the MHD force is defined only by the imaginary part of the conductivity. However, in real systems, both the real and imaginary parts of the conductivity play a role. Indeed, in the equivalent circuit of Fig. 1(a), both inductance and capacitance are represented by lumped elements, i.e., they are localized, resulting in a strict $\pi / 2$ phase difference between the electric and magnetic fields. On the other hand, in real systems, inductance and capacitance are distributed along the entire circuit, which affects the phase difference and allows a non-zero average force even with purely real conductivity. Nevertheless, for analytical considerations, we will treat the system as a circuit with lumped elements.

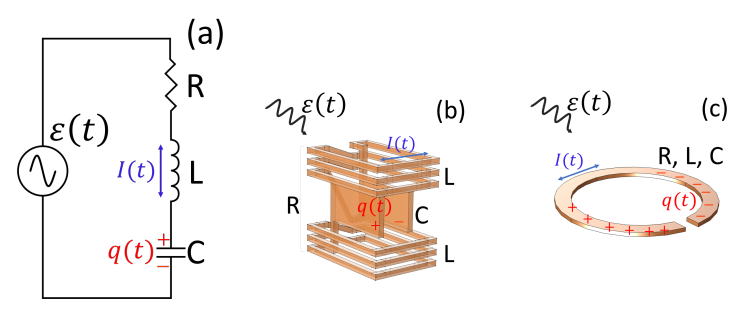

FIG. 1. (Color online) (a) Equivalent electric circuit of a particle, and possible designs of self-propelled particles: (b) particle with prominent inductance and capacitance, and (c) split-ring resonator.

Figures 1(b) and 1(c) show two possible particle designs, which can generate crossed electric and magnetic fields and thus create MHD thrust. The simplest way to implement an $L C$-circuit with crossed electric field from the capacitor and magnetic field from the inductor is represented by design (b). However, simulations showed that it has no efficiency advantage over design (c), which is a well-known split-ring resonator (SRR). Although it does not have such a distinct separation of capacitance and inductance, it can still be treated as an $L C$-circuit, and it produces crossed fields in the space outside of it. In addition, it is remarkably simpler and can be realized at different scales for different frequency bands. Therefore, in the following, we only focus on the analysis of design (c).

A sub-wavelength SRR can be characterized by its inductance $L$, capacitance $C$, and resistance $R$. The geometry of the problem is illustrated in Fig. 2(a). We assume that the SRR can be electrically polarized only in the $x_{0}$-direction and magnetically polarized only in the $y_{0}$-direction in the reference coordinate system attached to the SRR. The equation for the charge stored in the capacitor $q$ has the following form:

$$
\frac{\mathrm{d}^{2} q}{\mathrm{~d} t^{2}}+\Gamma \frac{\mathrm{d} q}{\mathrm{~d} t}+\omega_{0}^{2} q=\frac{1}{L} \varepsilon(t)
$$

where $\Gamma=R / L, \omega_{0}^{2}=1 / L C$, and $\varepsilon(t)$ is the effective EMF, which appears as a result of the interaction between the SRR and the electromagnetic wave. The EMF can be separated in two parts: that associated with the polarization of the SRR by the electric field and that attributed to the electromagnetic induction due to the variation of the magnetic field flux through the SRR:

$$
\begin{array}{r}
\varepsilon(t)=\varepsilon_{e}(t)+\varepsilon_{m}(t), \\
\varepsilon_{e}(t)=2 r_{0} a_{e} E_{\mathrm{ext}}(t) \cos \phi_{e}, \\
\varepsilon_{m}(t)=-\frac{\mathrm{d} \Phi}{\mathrm{d} t}=-i \omega \pi r_{0}^{2} B_{\mathrm{ext}}(t) \cos \phi_{m},
\end{array}
$$

where $r_{0}$ is the inner radius of the SRR, $a_{e}$ is some dimensionless constant of the order of $1, E_{\text {ext }}$ and $B_{\text {ext }}$ are the amplitudes of the electric and magnetic fields, respectively, and $\phi_{e}\left(\phi_{m}\right)$ 
is the angle between the electric (magnetic) field and the direction of the non-zero electric (magnetic) polarizability (see Fig. 2(a)). It should be noted that these angles are not independent. However, for Eqs. (8)-(11), having angles depend on each other is irrelevant.
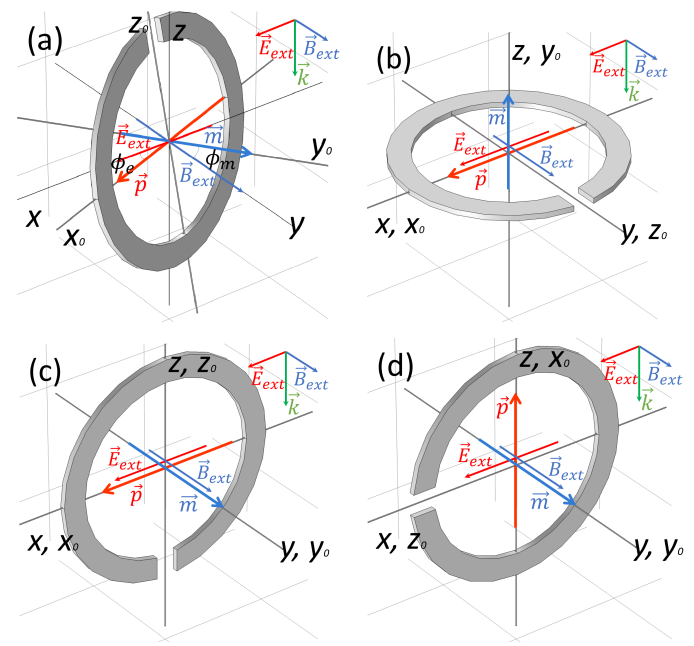

FIG. 2. (Color online) Orientation of the proposed active particle in an external field. (a) General orientation of an SRR and its reference coordinate system $\left(x_{0}, y_{0}, z_{0}\right)$ with respect to the fixed laboratory coordinate system $(x, y, z)$. (b) SRR orientation below the resonance with the electric dipole moment and the electric field aligned. (c) SRR orientation at the resonance with both dipole moments aligned with the corresponding fields. (d) SRR orientation above the resonance with the magnetic dipole moment and magnetic field aligned..

The angles $\phi_{e}$ and $\phi_{m}$ are defined by the orientation of the particle with respect to the external electric and magnetic fields of the electromagnetic wave. The equilibrium orientation of the SRR is governed by the minimum of its potential energy in the external fields. The potential energy can be expressed as:

$$
U=-\frac{1}{2} \mathbf{p}_{\omega} \mathbf{E}_{\mathrm{ext} \omega}^{*}-\frac{1}{2} \mathbf{m}_{\omega} \mathbf{B}_{\mathrm{ext} \omega}^{*}+c . c .,
$$

where $\mathbf{p}_{\omega}$ and $\mathbf{m}_{\omega}$ are the complex amplitudes of the electric and magnetic dipole moments, respectively, $\mathbf{E}_{\operatorname{ext} \omega}$ and $\mathbf{B}_{\operatorname{ext} \omega}$ are the complex amplitudes of the external electric and magnetic fields, respectively, and c.c. stands for 'complex conjugate.' The induced dipole moments can be found in terms of the electric charge in the capacitor. Namely,

$$
\mathbf{p}_{\omega}=\mathbf{x}_{0} 2 r_{0} a_{e} q_{\omega}
$$

and

$$
\mathbf{m}_{\omega}=-\mathbf{y}_{0} \pi r_{0}^{2} i \omega q_{\omega}
$$

where $\mathbf{x}_{0}$ and $\mathbf{y}_{0}$ are the unit vectors in the reference coordinate system attached to the SRR. The expression for $q_{\omega}$ can be found by solving Eqs. (8)-(11):

$$
q_{\omega}=S(\omega) C\left(2 r_{0} a_{e} E_{\mathrm{ext} \omega} \cos \phi_{e}-i \omega \pi r_{0}^{2} B_{\mathrm{ext} \omega} \cos \phi_{m}\right),
$$

where

$$
S(\omega)=\frac{\omega_{0}^{2}}{\omega_{0}^{2}+i \Gamma \omega-\omega^{2}}
$$

Finally, we obtain:

$$
\begin{array}{r}
U=\operatorname{Re}(S(\omega))\left[-\alpha_{e}\left|E_{\mathrm{ext} \omega}\right|^{2} \cos ^{2} \phi_{e}+\alpha_{m}\left|B_{\mathrm{ext} \omega}\right|^{2} \cos ^{2} \phi_{m}\right] \\
-\operatorname{Im}(S(\omega)) \chi_{e m} \cos \phi_{e} \cos \phi_{m} \operatorname{Re}\left(E_{\mathrm{ext} \omega} B_{\operatorname{ext} \omega}^{*}\right)
\end{array}
$$

where $\alpha_{e}=4 r_{0}^{2} a_{e}^{2}, \alpha_{m}=\pi^{2} r_{0}^{4} \omega^{2}$, and $\chi_{e m}=2 \pi r_{0}^{3} a_{e}$. The minimum of $U$ determines the equilibrium orientation of the particle in the field of an electromagnetic wave. When $\operatorname{Re}(S(\omega)) \gg \operatorname{Im}(S(\omega))$, it is easy to see that below resonance, i.e., for $\operatorname{Re}(S(\omega))>0$, the particle orients such that its electric dipole moment is parallel to the external electric field and its magnetic dipole moment is perpendicular to the external magnetic field (see Fig. 2(b)). On the other hand, above the resonance, i.e., for $(\operatorname{Re}(S(\omega))<0$, the electric dipole moment orients perpendicular to the external electric field, while the magnetic dipole moment aligns with the external magnetic field (see Fig. 2(d)). In the vicinity of the resonance, the orientation is governed by the imaginary part of $S(\omega)$. It can be shown that, in this case, both magnetic and electric dipole moments align with the corresponding fields (see Fig. 2(c)). Such a behavior can be explained by taking into account that, below resonance, the charge (and hence the electric dipole moment) oscillates in phase with the external field and the current (and hence the magnetic dipole moment) oscillates in opposite phase, while above resonance, conversely, the current oscillates in phase and the charge oscillates in opposite phase. It is noteworthy that unlike the MHD force, the orientational moments appear as a result of the direct action of the electromagnetic field on the particle. Therefore, the orientational motions are not coupled to the surrounding medium and are medium-independent.

As a result, the particle orientation can be controlled by the frequency and polarization of the electromagnetic wave. Indeed, the polarization specifies the directions of the electric and magnetic fields, while the frequency defines the orientation of the SRR with respect to them.

The consistent derivation of the equations of orientational motions is given in the Supplementary Material.

The particle experiences the action of several forces induced by the electromagnetic wave. These forces are schematically shown in Fig. 3. First, there is a reaction force coupled to the body force acting on the surrounding liquid. Second, there is a force caused by the radiation scattering off the particle. In the Supplementary Material, it is shown that the scattering force can be neglected in the current problem setting.

To find the MHD force, the integration of the force density over the volume (Eq. (5)) has to be performed. We approximate the excited SRR with crossed electric and magnetic dipoles, which oscillate with $\pi / 2$ phase difference, see Eqs. (13) and (14). Therefore, the scattered electric and magnetic 


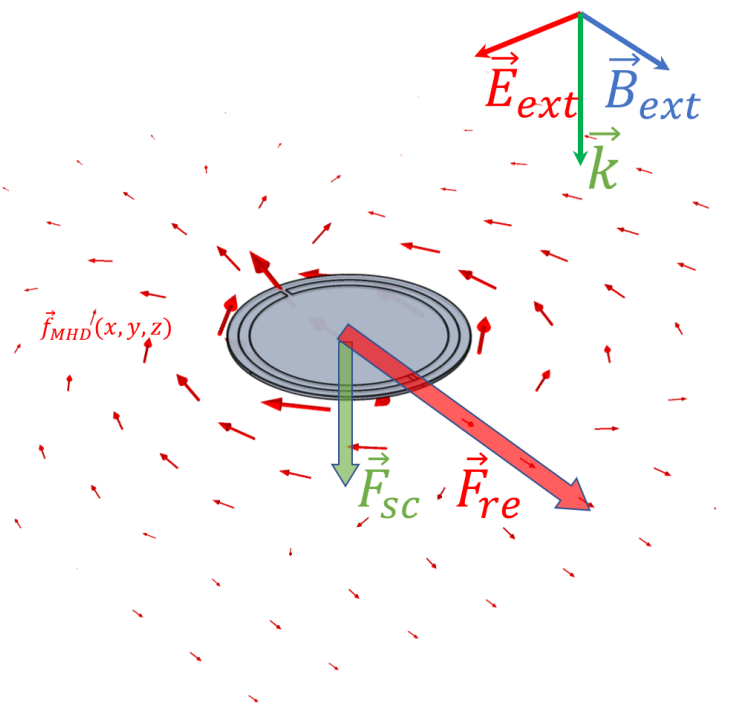

FIG. 3. (Color online) Schematic representation of the forces acting in the system: $\mathbf{f}_{M H D}(x, y, z)$ represents the body MHD force, $\mathbf{F}_{r e}$ represents the reaction force, and $\mathbf{F}_{s} c$ represents the scattering force.

fields can be found as:

$$
\begin{aligned}
& \mathbf{E}_{s c \omega}(r)=\frac{1}{4 \pi \varepsilon_{0}}\left(\frac{3 \mathbf{r}\left(\mathbf{p}_{\omega} \cdot \mathbf{r}\right)}{r^{5}}-\frac{\mathbf{p}_{\omega}}{r^{3}}\right), \\
& \mathbf{B}_{s c \omega}(r)=\frac{\mu_{0}}{4 \pi}\left(\frac{3 \mathbf{r}\left(\mathbf{m}_{\omega} \cdot \mathbf{r}\right)}{r^{5}}-\frac{\mathbf{m}_{\omega}}{r^{3}}\right),
\end{aligned}
$$

where $\mu_{0}$ is the vacuum permeability. We assume that the particle is oriented such that the electric dipole moment is along the $x$-axis and magnetic dipole moment is along the $y$-axis. By substituting Eqs. (18) and (19) into Eq. (5), after extensive but straightforward calculations, the expression for the MHD force can be obtained as follows:

$$
\mathbf{F}_{\mathrm{MHD}}=\mathbf{z}_{0} \operatorname{Re}\left[\frac{\sigma \mu_{0}}{4 \pi \varepsilon_{0}} \frac{p_{\mathrm{x} \omega} m_{\mathrm{y} \omega}^{*}}{3} \frac{1}{r_{m}^{3}}\right],
$$

where $\mathbf{z}_{0}$ is the third unit vector in the reference coordinate system attached to the particle and $r_{m}$ is some cut-off distance, below which the particles can no more be treated as dipoles. The reasonable cut-off distance may be chosen of the order of the particle radius, $r_{m}=\beta r_{0}$, where $\beta$ is a dimensionless constant of the order of 1 . It should be noted that the MHD force is defined solely by the scattered fields, i.e., the following integrals converge to zero:

$$
\begin{aligned}
& \int_{V} \operatorname{Re}\left(\sigma\left[\mathbf{E}_{\mathrm{ext} \omega} \times \mathbf{B}_{\mathrm{sc} \omega}^{*}\right]\right) d V=0, \\
& \int_{V} \operatorname{Re}\left(\sigma\left[\mathbf{E}_{\mathrm{sc} \omega} \times \mathbf{B}_{\operatorname{ext} \omega}^{*}\right]\right) d V=0 .
\end{aligned}
$$

Finally, substituting Eqs. (13) and (14) in Eq. (20) and assuming $\operatorname{Re}(S(\omega))>0$ and the corresponding particle orientation (Fig. 2(b)) for definiteness, we obtain:

$$
\mathbf{F}_{\mathrm{MHD}}=-\mathbf{z}_{0} \frac{2 \mu_{0} \operatorname{Im} \sigma}{3 \varepsilon_{0}}|S(\omega)|^{2} C^{2} \omega r_{0}^{2} E_{\text {ext } \omega}^{2} \frac{a_{e}^{3}}{\beta^{3}} .
$$

According to Eq. (20), the MHD force is always directed along the $z_{0}$-axis in the coordinate system attached to the particle. On the other hand, the orientation of the particle, and thus the direction of the $z_{0}$-axis in the fixed laboratory coordinate system, is defined by the minimum of the potential energy, Eq. (17), and hence the polarization and frequency of the electromagnetic wave. As a result, the direction of particle motion can be controlled by the polarization and frequency of the electromagnetic wave.

Electromagnetic simulations were performed using COMSOL Multiphysics to analyze the feasibility of the proposed particle concept. As was mentioned above, the sophisticated design of Fig. 1(b) did not show any advantages over the simple SRR design of Fig. 1(c), and it was thus disregarded.

The particle material was chosen as copper, and the surrounding liquid was chosen as water at room temperature. The electromagnetic properties of water were simulated with the Debye model $^{19}$. As the static conductivity is irrelevant for the desired effect, the ionic concentration was set to zero.

Figure 4 shows the frequency dependencies of the propulsion force for an SRR (Fig. 1(c)) illuminated by a plane electromagnetic wave for different particle sizes and two particle orientations. The radiation intensity was set to $4.8 \mathrm{~kW} / \mathrm{m}^{2}$. As can be seen, the peak force decreases by about a factor of 3 , from $F_{1} \approx 6 \times 10^{-13} \mathrm{~N}$ to $F_{7} \approx 2 \times 10^{-13} \mathrm{~N}$ in case (a) and from $F_{1} \approx 4.75 \times 10^{-13} \mathrm{~N}$ to $F_{7} \approx 1.6 \times 10^{-13} \mathrm{~N}$ in case (b), when the size of the particle decreases by a factor of 2 . As a result, the ratio of the peak force to the particle's own weight increases as the particle size decreases. Namely, this ratio increases from 0.23 for particle (1) to 0.68 for particle (7), all proportions of the particles being equal.

The comparison between the simulations and analytical solution Eq. (22) is given in the Supplementary Material.

The body force acting on the liquid around the particle allows to find the actual fluid flows. Examples of the flows around the particle are shown in the Supplementary Material.

The potential applicability of embedding the particles in a polimer matrix for structural integrity preservation and buoyancy compensation is also analyzed in the Supplementary Material.

In conclusion, we proposed self-propelled microparticles, which utilize the energy of an electromagnetic wave to create thrust. The electromagnetic wave causes a resonant excitation of crossed electric and magnetic scattered fields. The scattered fields produce the MHD force, which accelerates the surrounding fluid and, in response, propels the particle. Theoretical estimates as well as simulations show that the MHD thrust is comparable to the weight of the particle itself for particles of the order of $10 \mu \mathrm{m}$ in size. The particle orientation can be controlled by the frequency and polarization of the radiation. In addition, the proposed particles can be embedded in a dielectric matrix for structural robustness and gravity compensation without significant loss of thrust. 

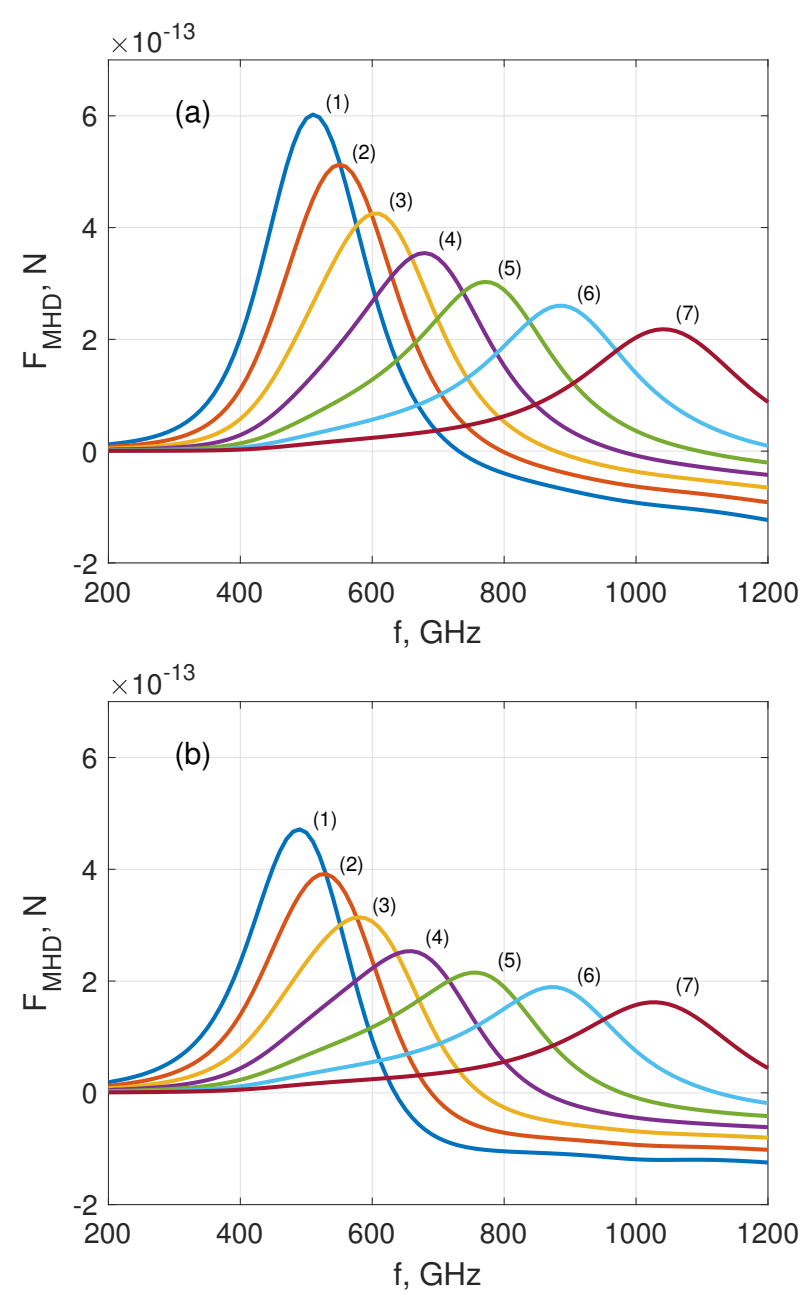

FIG. 4. (Color online) MHD propulsion force as a function of radiation frequency for different particle sizes and two orientations: the particle is oriented such that (a) the electric dipole moment is parallel to the external electric field, and the magnetic dipole moment is perpendicular to the external magnetic field (see Fig. 2(b)); and (b) the electric dipole moment is perpendicular to the external electric field, and the magnetic dipole moment is parallel to the external magnetic field (see Fig. 2(d)). The particles were illuminated by a linearly polarized plane wave with the intensity of $4.8 \mathrm{~kW} / \mathrm{m}^{2}$. The particle sizes are as follows: (1) $r_{0}=16.8 \mu \mathrm{m}$; (2) $r_{0}=15.4 \mu \mathrm{m}$; (3) $r_{0}=14.0 \mu \mathrm{m}$; (4) $r_{0}=12.6 \mu \mathrm{m}$; (5) $r_{0}=11.2 \mu \mathrm{m}$; (6) $r_{0}=9.8$ $\mu \mathrm{m} ;$ (7) $r_{0}=8.4 \mu \mathrm{m}$.

\section{SUPPLEMENTARY MATERIAL}

Supplementary material contains the derivation of the equations of rotational motion, comparison between the scattering and MHD forces, comparison between simulation and analytics, discussion on the possibility of embedding the particles in matrix, and examples of fluid flows around the particle.

\section{ACKNOWLEDGMENTS}

This study was supported by the French PIA project "Lorraine Université d'Excellence", reference ANR-15-IDEX-04LUE and TALiSMAN project, funded by FEDER (2019000214).

\section{SUPPORTING DATA}

The data that support the findings of this study are available from the corresponding author upon reasonable request.

${ }^{1}$ S. Ramaswamy, “Active matter,” J. Stat. Mech. 26, 054002 (2017).

${ }^{2}$ M. C. Marchetti, J. F. Joanny, S. Ramaswamy, T. B. Liverpool, J. Prost, M. Rao, and R. A. Simha, "Hydrodynamics of soft active matter," Rev. Mod. Phys. 85, 1143 (2013).

${ }^{3}$ M. R. Shaebani, A. Wysocki, R. G. Winkler, G. Gompper, and H. Rieger, "Computational models for active matter," Nat Rev Phys 2, 181 (2020).

${ }^{4}$ C. Bechinger, R. Di Leonardo, H. L?wen, C. Reichhardt, G. Volpe, and G. Volpe, "Active particles in complex and crowded environments," Rev. Mod. Phys. 88, 045006 (2016).

${ }^{5}$ J. Elgeti, R. G. Winkler, and G. Gompper, "Physics of microswimmers - single particle motion and collective behavior: a review," Rep. Prog. Phys. 78, 056601 (2015).

${ }^{6}$ K. Ishimoto, H. Gad?ha, E. A. Gaffney, D. J. Smith, and J. Jackson Kirkman-Brown, "Coarse-graining the fluid flow around a human sperm," Phys. Rev. Lett. 118, 124501 (2017).

${ }^{7}$ W. F. Paxton, K. C. Kistler, C. C. Olmeda, A. Sen, S. K. St Angelo, Y. Cao, T. E. Mallouk, P. E. Lammert, and V. H. Crespi, "Catalytic nanomotors: autonomous movement of striped nanorods," J. Am. Chem. Soc. 126, 13424 (2004).

${ }^{8}$ J. R. Howse, R. A. L. Jones, A. J. Ryan, T. Gough, R. Vafabakhsh, and R. Golestanian, "Self-motile colloidal particles: From directed propulsion to random walk," Phys. Rev. Lett. 99, 048102 (2007).

${ }^{9}$ W. Wang, W. Duan, S. Ahmed, T. E. Mallouk, and A. Sen, "Small power: autonomous nano- and micromotors propelled by self-generated gradients," Nano Today 8, 531 (2013).

${ }^{10}$ R. Golestanian, T. B. Liverpool, and A. Ajdari, "Propulsion of a molecular machine by asymmetric distribution of reaction products," Phys. Rev. Lett. 94, 220801 (2005).

${ }^{11}$ R. Golestanian, T. B. Liverpool, and A. Ajdari, "Designing phoretic microand nano- swimmers," New J. Phys. 9, 126 (2007).

${ }^{12}$ H.-R. Jiang, H. Wada, N. Yoshinaga, and M. Sano, "Manipulation of colloids by a nonequilibrium depletion force in a temperature gradient," Phys. Rev. Lett. 102, 208301 (2009).

${ }^{13}$ J. R. Jiang, N. Yoshinaga, and M. Sano, "Active motion of a Janus particle by self-thermophoresis in a defocused laser beam," Phys. Rev. Lett. 105, 268302 (2010).

${ }^{14}$ I. G. Buttinoni, G. Volpe, F. K?mmel, G. Volpe, and C. Bechinger, "Active Brownian motion tunable by light," J. Phys. Condens. Matter 24, 284129 (2012).

${ }^{15} \mathrm{~A}$. Ghosh, and P. Fischer, "Controlled propulsion of artificial magnetic nanostructured propellers," Nano Lett. 9, 2243 (2009).

${ }^{16}$ P. Tierno, R. Golestanian, I. Pagonabarraga, and F. Sagu?s, "Controlled swimming in confined fluids of magnetically actuated colloidal rotors," Phys. Rev. Lett. 101, 218304 (2008).

${ }^{17}$ R. Dreyfus, J. Baudry, M. L. Roper, M. Fermigier, H. A. Stone, and J. Bibette, "Microscopic artificial swimmers," Nature 437, 862 (2005).

${ }^{18}$ L. D. Landau, E. M. Lifshitz, L.P. Pitaevskii, Electrodynamics of Continuous Media. Vol. 8, (2nd ed.), Butterworth-Heinemann, Oxford 1984.

${ }^{19}$ W. Ellison, "Permittivity of pure water, at standard atmospheric pressure, over the frequency range 0-25 thz and the temperature range 0-100 c," J. Phys. Chem. Ref. Data 36, 1 (2007).

${ }^{20}$ O. Sydoruk, E. Tatartschuk, E. Shamonina, and L. Solymar, "Analytical formulation for the resonant frequency of split rings," J. Appl. Phys. 105, 014903 (2009). 


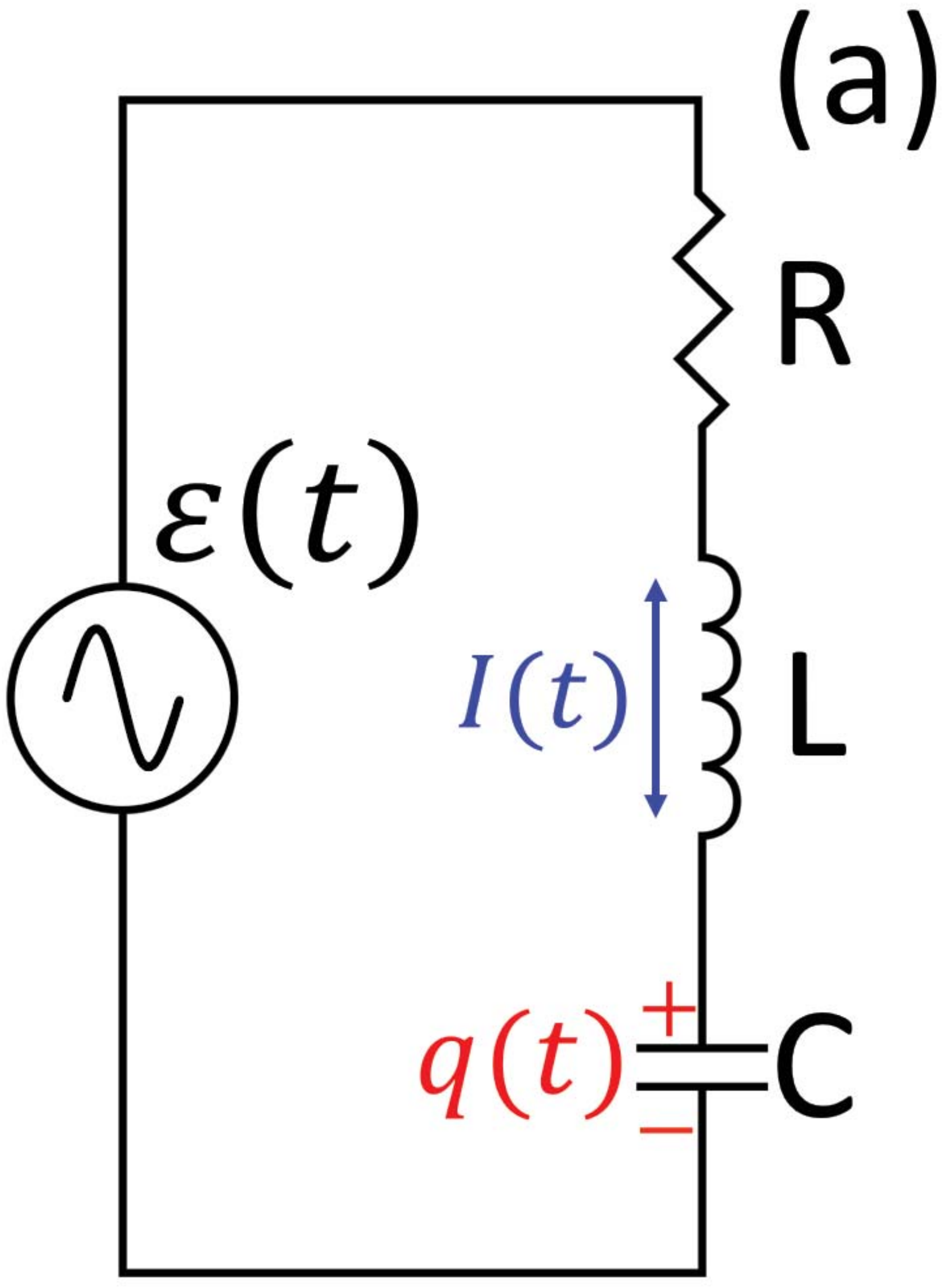


$\eta^{\varepsilon(t)}$

(b)

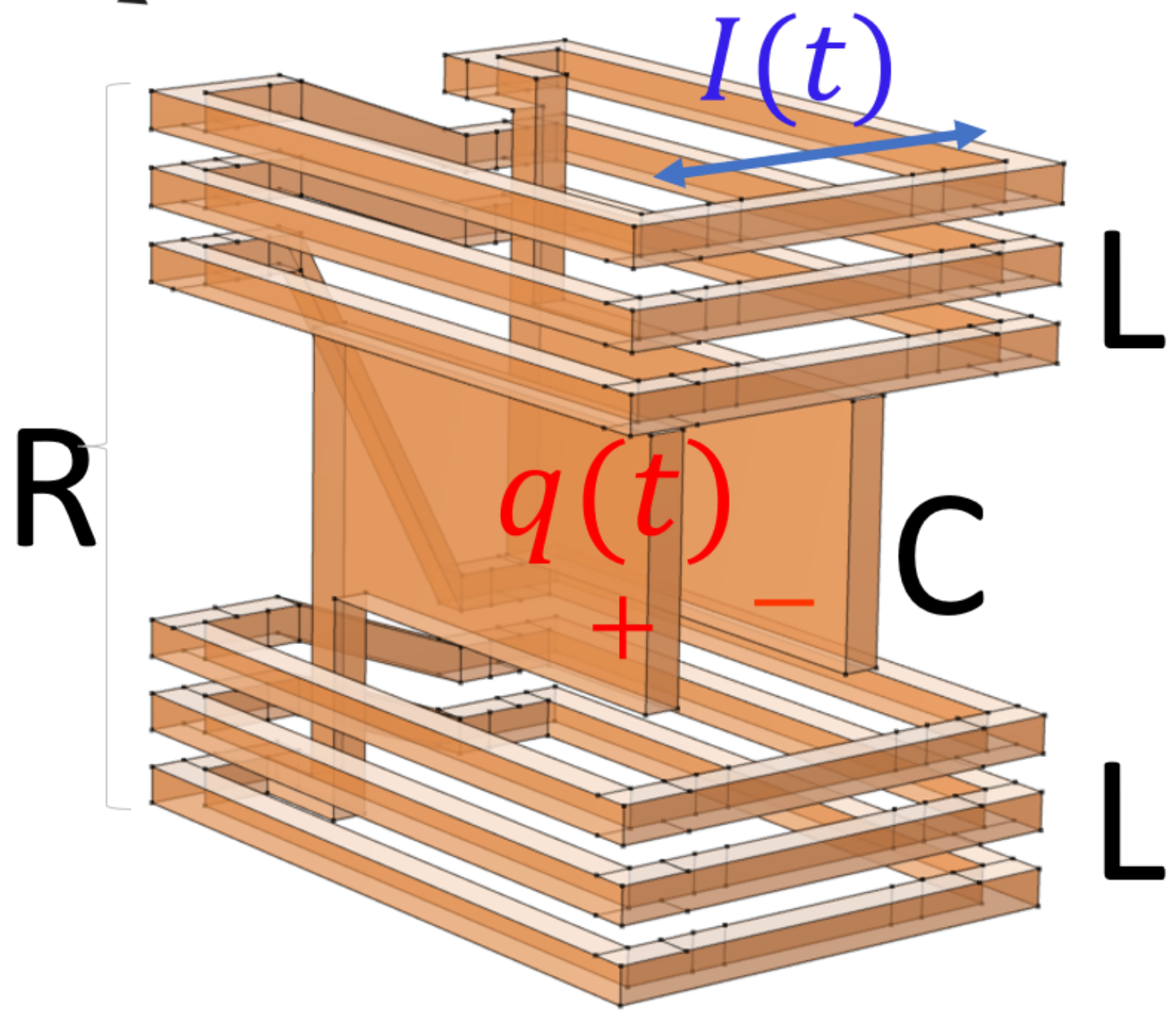


$\eta^{\varepsilon(t)}$
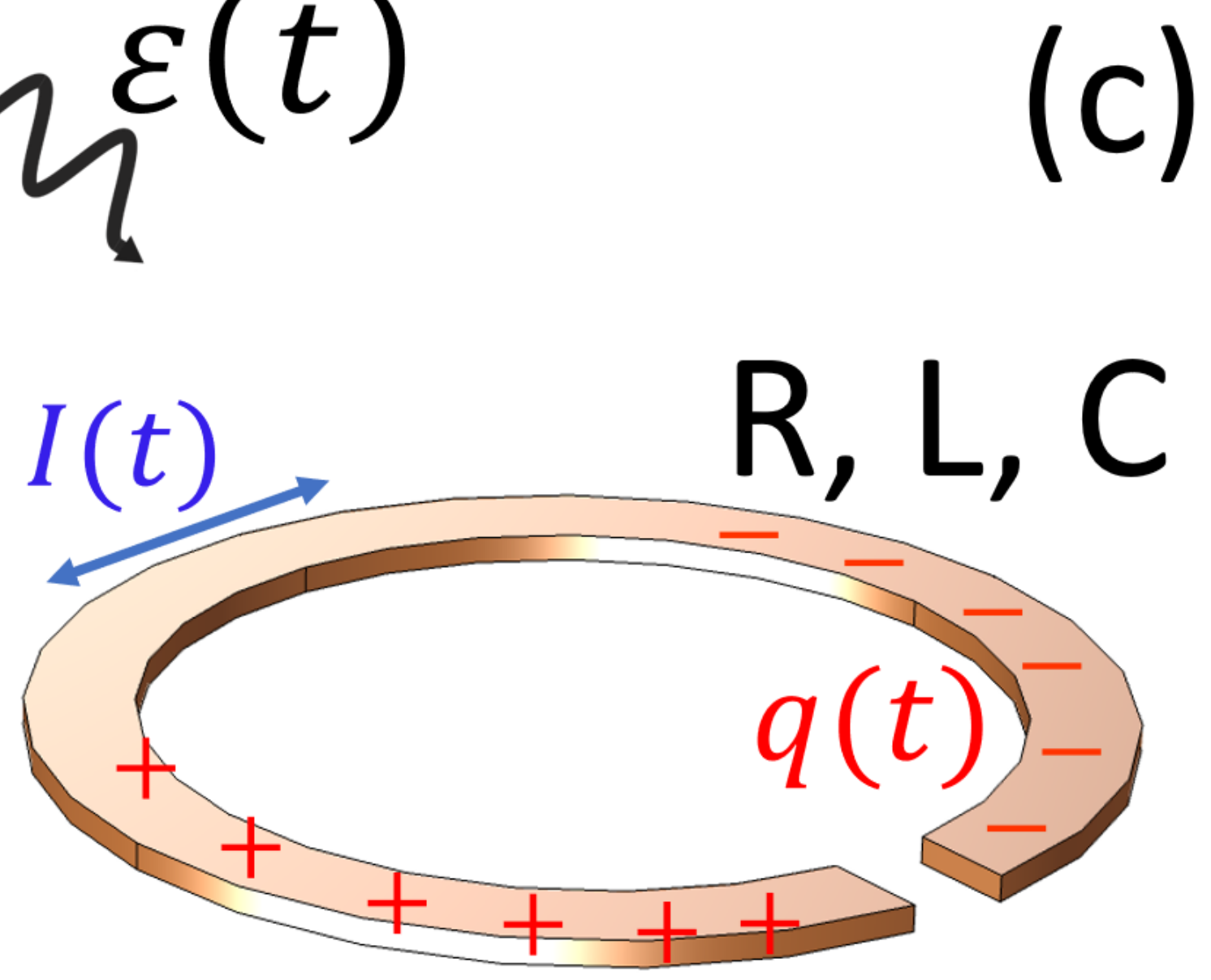


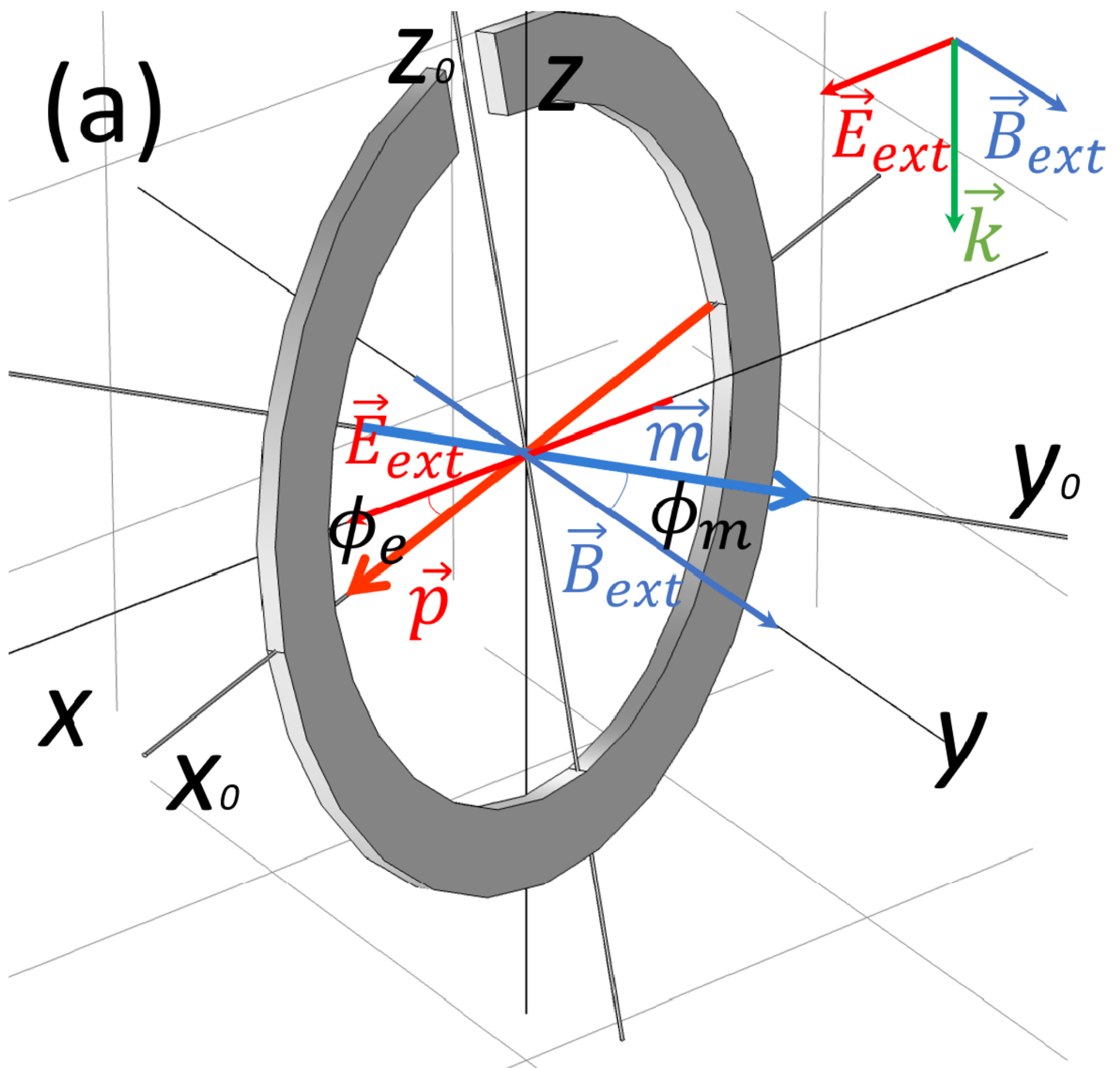




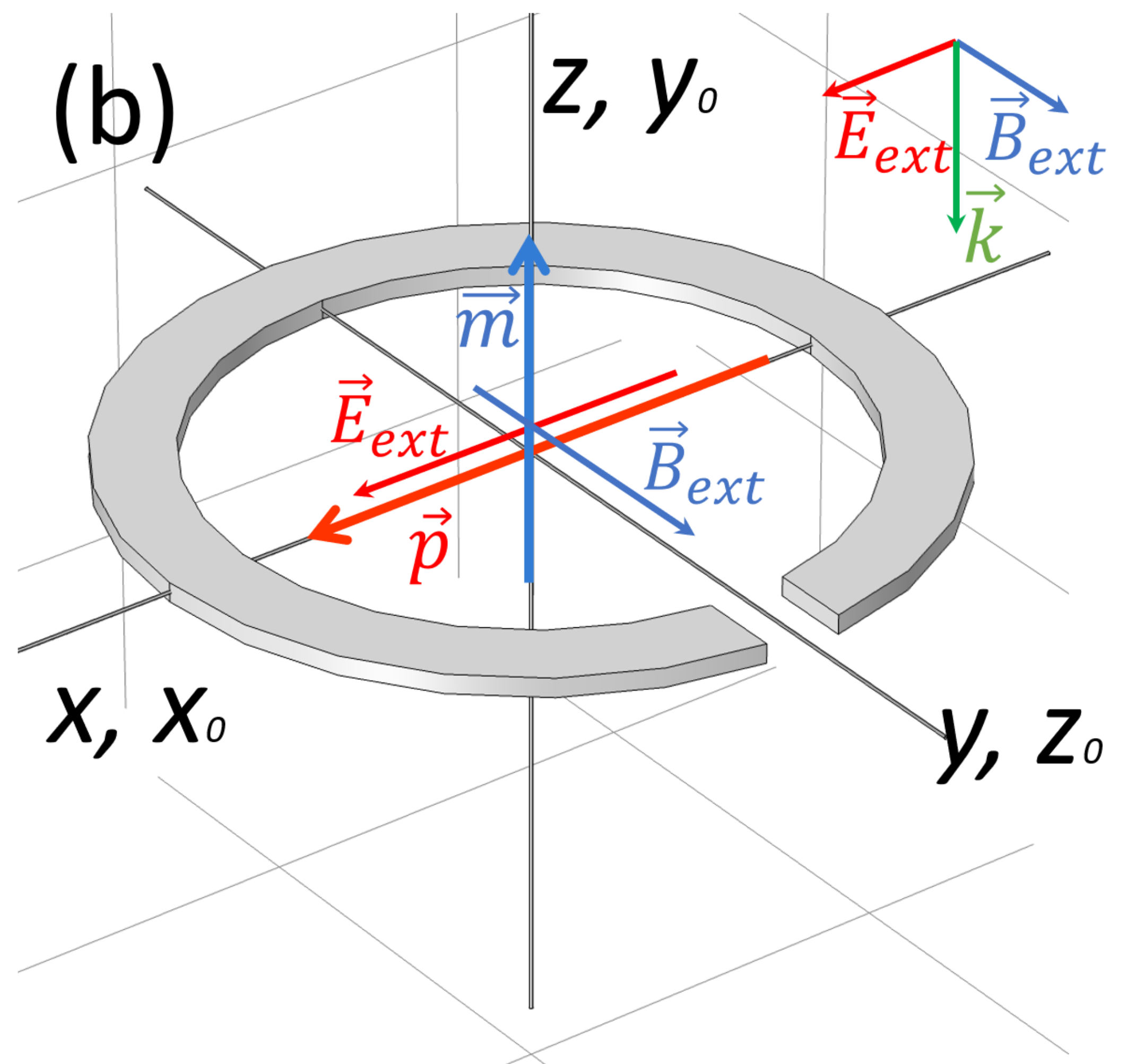




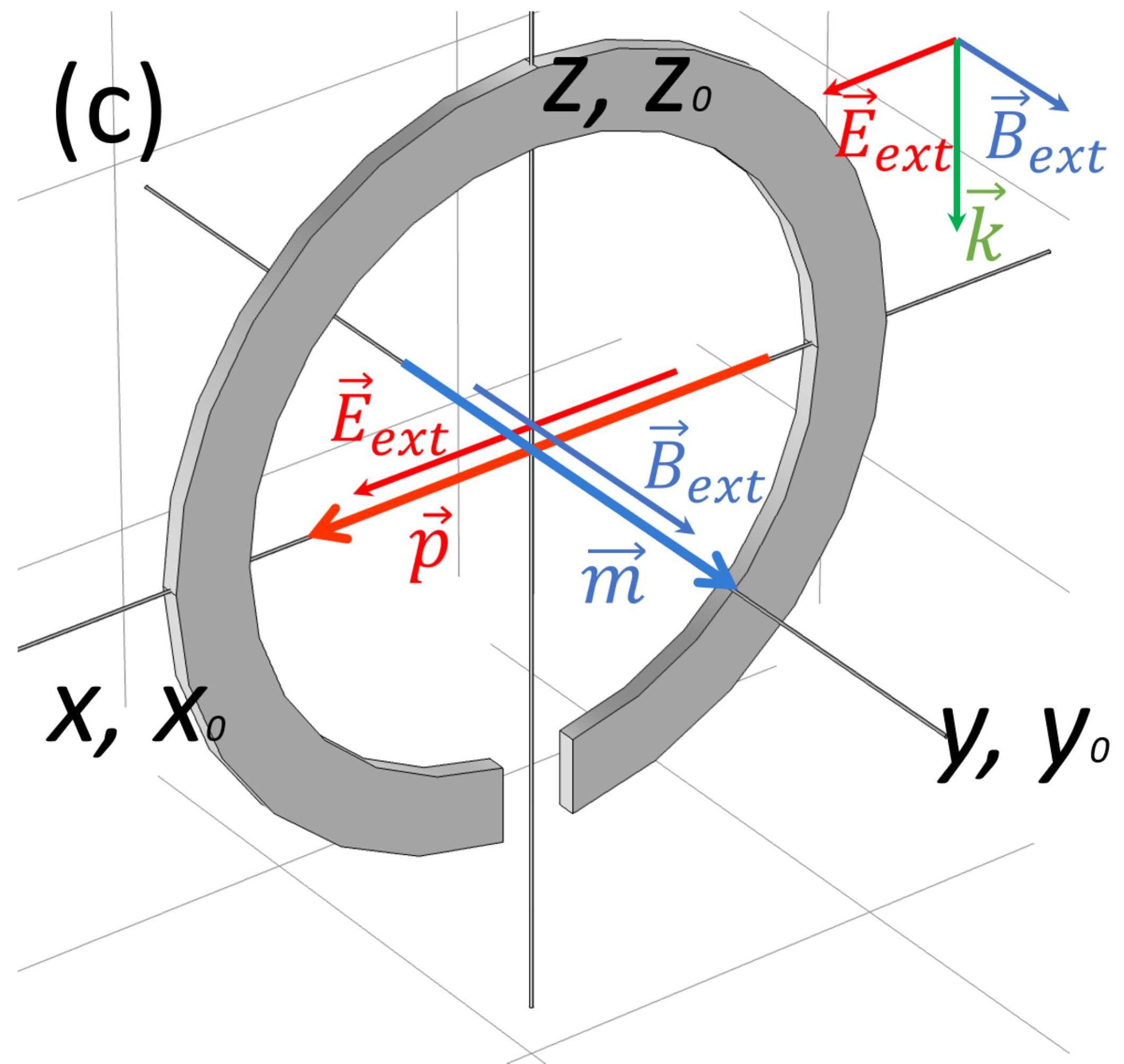




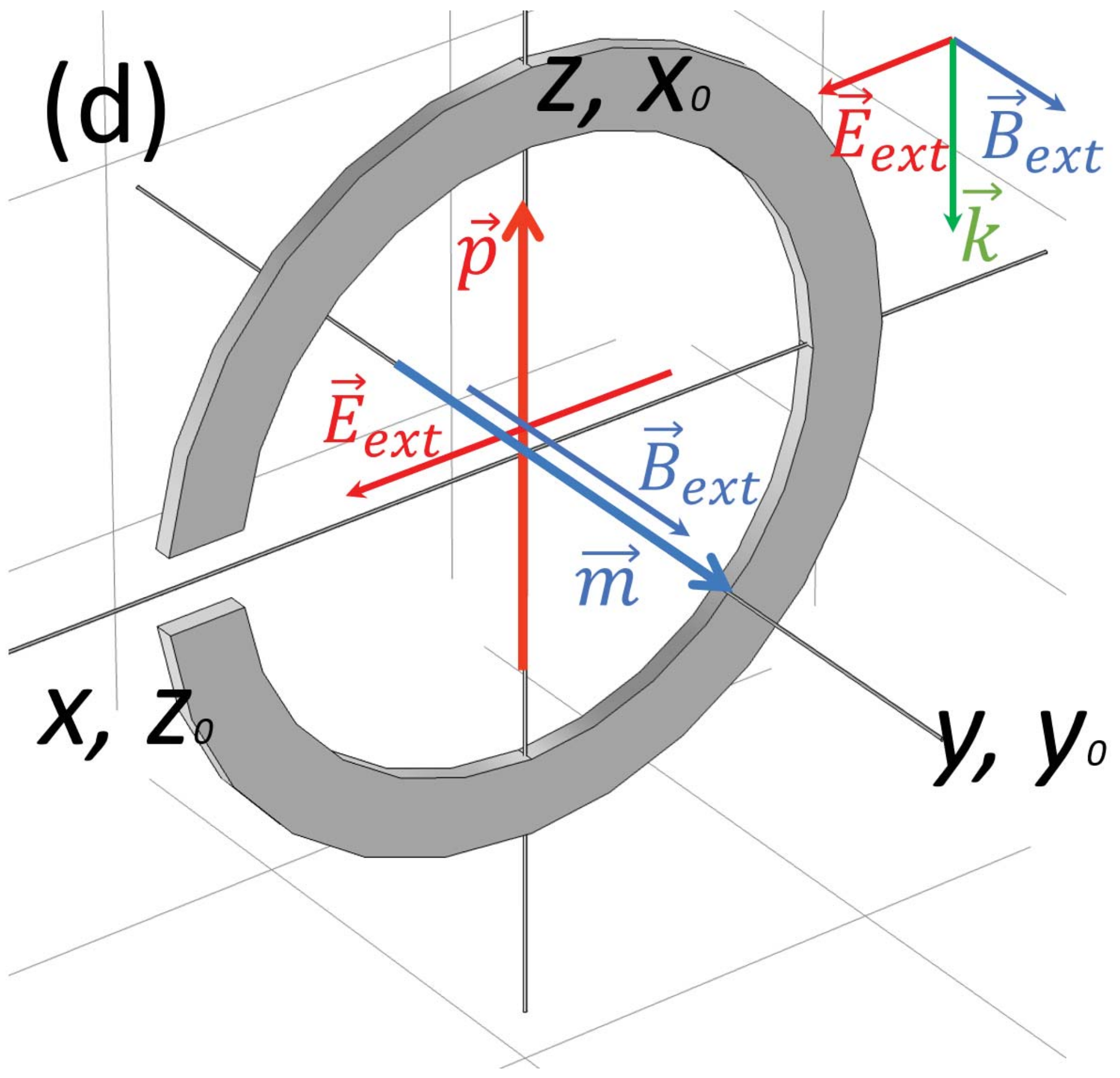




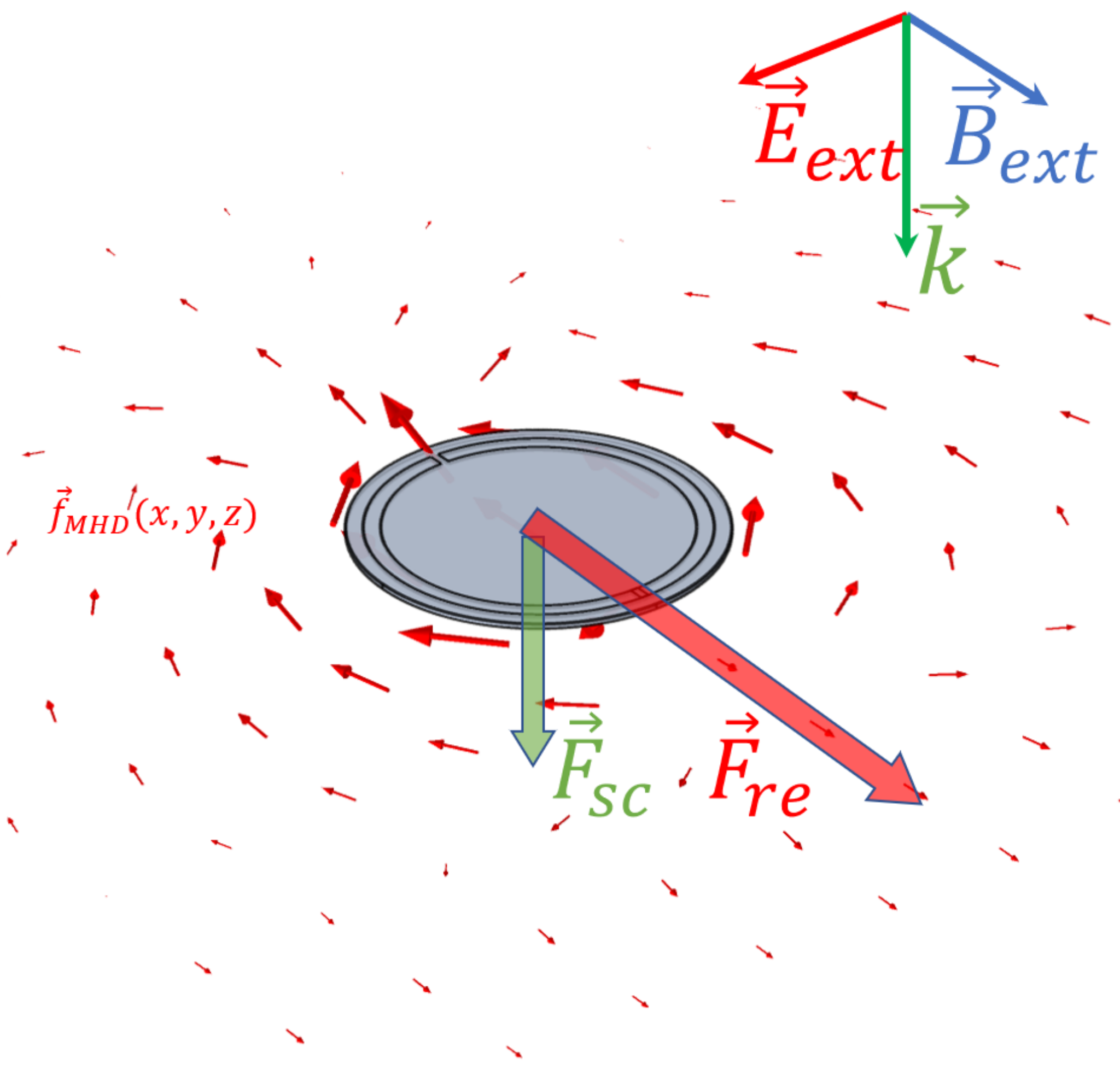




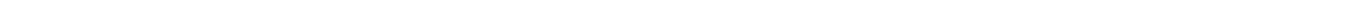




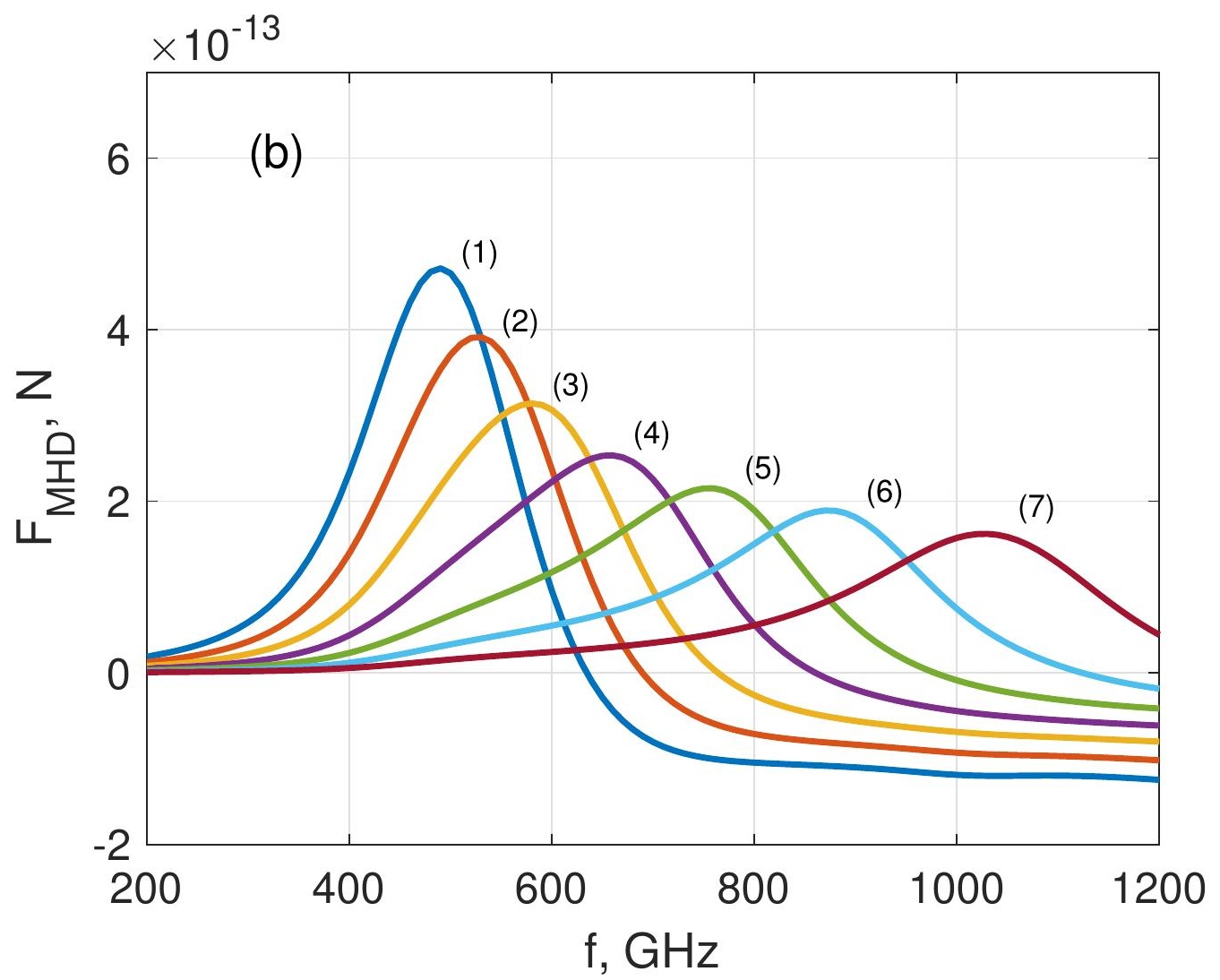

